\title{
AfnP-Vorstandswahlen 2015-2019 und Mitgliederversammlung Vorstand wurde im Amt bestätigt
}

Am 27.11.2015 wählten die AfnP-Mitglieder ihren neuen Vorstand für die nächsten 4 Jahre. Marion Bundschu wurde in ihrem Amt bestätigt. Ebenso bleibt Hans-Martin Schröder stellvertretender Vorsitzender. Schriftführerin ist Ilona Adler und Schatzmeisterin Roswitha Nicklas. Zum beratenden Beirat wurde Rebecca Fiedler ernannt.

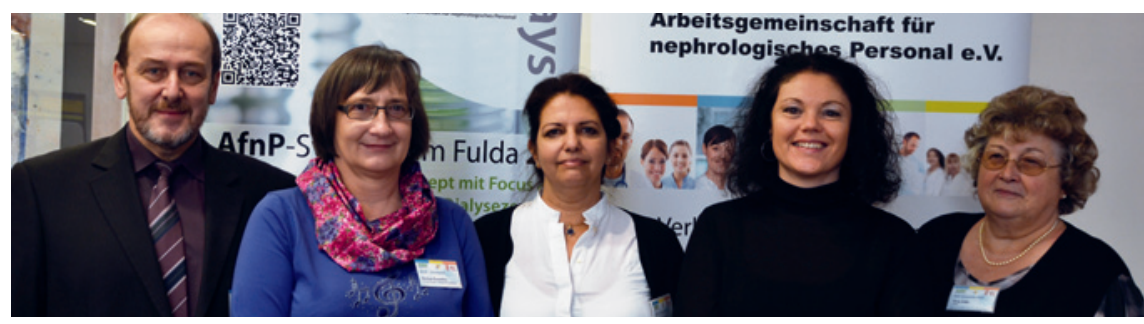

Abb. 1 Der neue Vorstand und beratende Beirat der AfnP e. V. (von links): Hans-Martin Schröder, Roswitha Nicklas, Marion Bundschu, Rebecca Fiedler und Ilona Adler.

Am 27.11.2015 wurde der neue (alte) AfnP-Vorstand für den Zeitraum 20152019 in einer geheimen Wahl gewählt. Er wird im Folgenden vorgestellt.

\section{AfnP-Vorstand}

\section{Marion Bundschu 1. Vorsitzende}

Als 1. Vorsitzende der AfnP e.V. wurde Marion Bundschu gewählt (Abb. 1). Seit 1980 ist sie Mitglied in der AfnP, seit 1986 aktiv in der AfnP als Ländervertreterin Baden-Württemberg und seit 2008 AfnPVorstandsvorsitzende.

Viele Jahre ist sie nun schon aktiv oder beratend in den verschiedenen Arbeitskreisen tätig, beispielsweise der Bundesarbeitsgemeinschaft der Weiterbildungsstätten, der Arbeitsgruppe der deutschen Krankenhausgesellschaft und der Hygienearbeitsgruppe. Seit 2006 kümmert sie sich aktiv um das Programm des Symposiums sowie der Dialyse Basic Days (DBD) in Fulda und die dazu notwendige Organisation.

2012 konnte die BANP (Bundesarbeitsgemeinschaft Nephrologische Pflege) als gemeinsames Sprachrohr für den berufspolitischen Dialog mit den Fachgesellschaften ins Leben gerufen werden. Bezahlbare Fort- und Weiterbildungen für alle nephrologisch tätigen Berufsgruppen ist ihr eine Herzensangelegenheit. Die Nephro Fachtagung Ulm (www.nephroulm.de) als Kooperation zwischen der AfnP e.V., der Weiterbildungsstätte Ulm (www.wbs-ulm.de) und dem Georg Thieme Verlag, ist jetzt eine jährlich statt- findende etablierte Veranstaltung. Durch die Erweiterung des Vorstandes und einige junge Ländervertreter wird Frau Bundschu in den nächsten Jahren ihr Wissen und ihre langjährige Erfahrung Stück für Stück übertragen können.

Hans-Martin Schröder stellvertretender Vorsitzender

Als stellvertretender Vorsitzender wurde Hans-Martin Schröder gewählt (Abb.1). Hans-Martin Schröder ist seit 1984 AfnPLändervertreter in Rheinland-Pfalz und gehört seit 2004 dem Vorstand an. Er bekleidete zunächst das Amt des Schriftführers und ist seit 2007 2. Vorsitzender der AfnP. Er ist ein Gründungsmitglied der BANP. Infolge seines beruflichen Werdeganges ist er ein Allrounder und somit für die AfnP in den verschiedeen Bereichen (Technik, Pflege etc.) unverzichtbar.

\section{Roswitha Nicklas Schatzmeisterin}

Zur Schatzmeisterin wurde Roswitha Nicklas gewählt (Abb. 1). Seit 2014 ist sie kommissarisches Vorstandsmitglied als Schatzmeisterin und zuständig für die Mitgliederverwaltung. Als langjährig tätige Arzthelferin vertritt sie besonders die Interessen ihrer Berufsgruppe in der AfnP.

\section{Ilona Adler}

\section{Schriftführerin}

Zur Schriftführerin wurde Ilona Adler gewählt (Abb. 1). Von 1986-1990 war sie außerordentliches Mitglied in der Nephrologischen Gesellschaft der DDR. Sie ist bereits seit über 25 Jahren in der AfnP aktiv. Seit 1990 arbeitet sie aktiv im engeren Vorstand mit sowie als Ländervertreterin Sachsen/Sachsen-Anhalt. Sie wurde 1988 Fachschwester für Dialyse und Nierentransplantation und arbeitete viele Jahre im Dialysezentrum Görlitz. Im März 2014 wurde sie als kommissarisches Vorstandsmitglied zur Schriftführerin gewählt. Auch als „Rentnerin im Unruhestand“ möchte sie in der ehrenamtlichen Tätigkeit unseren jungen Leuten ihre Erfahrungen vermitteln und mit Rat und Tat zur Seite stehen.

\section{Rebecca Fiedler beratender Beirat}

Zum beratenden Beirat des Vorstandes wurde Rebecca Fiedler ernannt (Abb. 1). Sie ist seit 2011 AfnP-Mitglied und Ländervertreterin für Hessen. Seit 2000 ist sie im Klinikum Fulda auf der Dialysestation tätig. 2007 machte sie eine Weiterbildung zur Praxisanleiterin und 2011-2013 eine Weiterbildung an der WBS-Ulm zur Fachschwester für Nephrologie und Dialyse.

Frau Fiedler kümmert sich seit einiger Zeit um den Auftritt der AfnP bei Facebook und möchte dies auch weiterhin gerne fortsetzen. Ihre Ziele sind, die AfnP weiterhin bei ihren Aufgaben zu unterstützen, wobei ihr besonderes Augenmerk bei den Veranstaltungen der AfnP liegen (Symposium, Dialyse Basic Days und Nephro Fachtagung Ulm).

\section{Ziele für die nächsten Jahre}

Die AfnP versteht sich als Verein für alle nephrologischen Berufsgruppen, egal ob examinierte Pflegekraft, MFA, Techniker, Arzt etc. Unser Ziel ist es, die bestehenden Kooperationen zu pflegen und neue aufzubauen. Weiter ist es uns ein besonderes Anliegen, bezahlbare Fortbildungen, Seminare und Tagungen in regelmäßigen Zeiträumen anzubieten. Dabei sind wir auf Ihre Wünsche und Anregungen angewiesen, um die Programmgestaltung an Ihre Anforderungen anzupassen. Unser Programm gestaltet der Programmbeirat und wählt Referenten aus, die nicht im finanziellen Interessenskonflikt stehen. Aus diesem Grund haben wir uns schon vor Jahren für die Zusammenarbeit mit dem Georg Thieme Verlag entschieden und dies nicht bereut. Mit einem Organschaftsvertrag bieten wir unseren Mitgliedern 10-mal im Jahr die Zusendung einer wissenschaftlich renommierten Zeitschrift - der Dialyse aktuell. Ärzte können CME-Punkte und Pflegekräfte Registrierungspunkte erwerben. 
Da die finanziellen Mittel bei einem Jahresbeitrag von 30 Euro ohne Sponsoren gering sind, wird unsere ehrenamtliche Arbeit stark beschränkt. Trotz des Einbringens von Freizeit und des Verzichts auf Vergütung des Arbeitseinsatzes, lässt sich so nicht alles umsetzen, wie wir es uns für unsere Mitglieder wünschen.

Seit 2012 bieten wir im Rahmen des AfnP-Symposiums das „Forum Technik“ an. Ziel ist es, in Themenschwerpunkten technische sowie gesetzliche Grundlagen, Basiswissen und neue Entwicklungen vorzustellen. 2014 sind wir mit den Dialyse Basic Days (DBD) sehr erfolgreich gestartet und werden die Veranstaltung 2016 wegen des guten Zuspruchs um einen halben Tag verlängern. Mit den DBD bieten wir zusätzlich zur Einarbeitung vertiefendes theoretisches und in der Anwenderschulung auch praktisches Wissen für alle an. Damit sollen Anwenderfehler verringert werden, woraus eine höhere Patientensicherheit resultiert. Zusätzlich bieten wir regionale Veranstaltungen über unsere aktiven Ländervertreter an. Die Zusammensetzung des multidisziplinären therapeutischen Teams verändert sich, bedingt durch ökonomische Zwänge und damit auch der Fort- und Weiterbildungsbedarf. Die AfnP reagiert darauf und möchte, dass neue und junge Mitarbeiter von den langjährig erfahrenen Kräften lernen sowie ihren Platz im Team finden. Damit auch zu Hause die Vorträge noch einmal nachgelesen werden können, bieten wir für die Teilnehmer einen Login zu den Präsentationen an.

Einen breiten Raum nimmt der Dialog untereinander sowie mit den Referenten ein. Der Blick über den Tellerrand ist für alle Teilnehmer eine Bereicherung. Die jährliche Nephro Fachtagung Ulm (26.-27.02.2016, Hotel Seligweiler, www.nephro-ulm.de) ist eine Kooperation zwischen der AfnP, der Weiterbildungsstätte Ulm sowie dem Georg Thieme Verlag und erfreut sich eines Zulaufs aus dem gesamten deutschsprachigen Raum. Diese Veranstaltung ist von der deutschen Registrierungsstelle für beruflich Pflegende und vom österreichischen Pflegeverband anerkannt.

Der nephrologische Arbeitskreis SaarPfalz-Mosel veranstaltet jährlich zusammen mit der AfnP eine Tagung. Ein weiteres gemeinsames Projekt mit dem Georg Thieme Verlag ist die jährliche Verleihung des Förderpreis Nephrologische Pflege. Der Preisträger erhält ein Preisgeld von 1500 Euro, welches vom Georg Thieme
Verlag (Herausgeber der Zeitschrift Dialyse aktuell) gestiftet wird.

\section{AfnP-Mitgliederversammlung}

Im Folgenden finden Sie einen Bericht zur AfnP-Mitgliederversammlung (MV) vom Samstag, den 28.11.2015, im Hotel Maritim Fulda (Beginn: 12:30 Uhr).

- zu TOP 1: Es wurde die satzungsgemäße Einladung und die Beschlussfähigkeit der MV 2015 festgestellt.

- zu TOP 2: Das Protokoll der MV vom 27.09.2014 wurde, nach nochmaligen Verlesen, einstimmig verabschiedet.

- zu TOP 3: Im Bericht des Vorstandes wurde auf die unterschiedlichsten Aktivitäten im vergangenen Jahr, hingewiesen. Das ehrenamtliche Engagement der Ländervertreter und des Vorstandes ist wieder besonders hervorzuheben. Die gute Zusammenarbeit mit dem Georg Thieme Verlag machte es wieder möglich, den mit 1500 Euro ausgelobten Förderpreis Nephrologische Pflege in diesem Jahr an 3 Preisträger zu verleihen.

- zu TOP 4: Bericht des Schatzmeisters über das Rechnungsjahr 2014: Der Stand über Einnahmen, Ausgaben und der Kontostand weisen auf einen ausgeglichenen Haushalt hin.

- zu TOP 5: Kassenprüfer Siegfried Tijunelis berichtete von einer ordnungsgemäß durchgeführten Kassenprüfung am 09.05.2015. Die Prüfung durch ihn und Jürgen Brunner ergab keinerlei Beanstandungen.

- zu TOP 6: Antrag auf Entlastung des alten Vorstandes: Der Vorstand wurde entlastet (Zustimmung und 3 Enthaltungen).

- zu TOP 7: Dankenswerterweise haben sich Jürgen Brunner und Siegfried Tijunelis für das Rechnungsjahr 2015 als Kassenprüfer zur Verfügung gestellt (einstimming angenommen).

- zu TOP 8: In diesem Jahr fanden wieder unsere Vorstandswahlen statt. Wie zu erwarten, ist der „alte“ Vorstand auch der neu gewählte: 1 . Vorsitzende Marion Bundschu (90\% der abgegebenen Stimmen), stellv. Vorsitzender Hans-Martin Schröder (92,5\% der abgeg. Stimmen), Schatzmeisterin Roswitha Nicklas (92,5\% der abgeg. Stimmen), Schriftführerin Ilona Adler (95\% der abgeg. Stimmen)

- zu TOP 9: Aus Gesundheits- und Altersgründen bat Siegfried Tijunelis um seine Verabschiedung. Nur ungern kamen wir seinem Wunsch nach. Mit seiner netten und unkomplizierten Art stand er uns nach seiner Verabschiedung in den „Rentner- und Unruhestand“ noch als Moderator und auch Mitorganisator von regionalen Fortbildungen zur Verfügung. Vielen Dank, Siegfried - wir wünschen dir und deiner Christel noch viele gemeinsame Jahre bei guter Gesundheit! Ein herzlicher Dank ging auch an Ralf Sachs. Er hat sich uns für Technikund Computerfragen während des Symposiums zur Verfügung gestellt.

- zu TOP 10: Antrag Satzungsanpassung auf Grundlage eines Schreibens des Finanzamts Ulm, um die Gemeinnützigkeit zu behalten, vom 05.11 .15 (einstimmig angenommen). Wortlaut der Anpassung: „§ 4 Rechte und Pflichten der Mitglieder: [...] (4) Jedes Mitglied erhält regelmäßig Zeitschriften aus den Verlagen, aus denen notwendige Informationen über die Vereinsaktivitäten sowie satzungsmäßige Themen entnommen werden können. Mit den Verlagen hat die AfnP einen Organschaftsvertrag. Weitere Information sind über die Homepage www.afnp.de und die Einwahl in den mitgliedergeschützten Bereich möglich. [...] § 8 Der Vorstand: [...] (11) Die Mitglieder des Vorstands arbeiten ehrenamtlich. Ihnen stehen jedoch für die Teilnahme an den Vorstandssitzungen, Treffen des Vorstands und Arbeitstreffen eine Erstattung der Reisekosten und eine Sitzungspauschale zu. Die Höhe einer Sitzungspauschale wird in der Ländervertreterversammlung festgelegt, ebenso die Kilometerpauschale bei der Anreise mit dem Auto. Für die Mitglieder besteht die Möglichkeit, für vereinsbezogene Tätigkeiten eine i.S. des $§ 55$ (1) AO angemessene Vergütung zu erhalten. Dies gilt auch für Vorstandsmitglieder. Über die Höhe der Vergütung entscheidet der Vorstand. Über die Höhe der Vergütung von Vorstandsmitgliedern entscheidet die LV-Versammlung.“

- zu TOP 11: Den anwesenden Mitgliedern wurde ein Ausblick auf die uns schon bekannten Termine 2016 gegeben. Auch auf die möglichen Tagungsortwechsel wurde hingewiesen. Nachlesen kann man das zeitnah auf unserer Homepage (www.afnp.de).

- zu TOP 12: Da dem Vorstand keine weiteren Anträge vorgelegt wurden, konnte die Mitgliederversammlung um 12:55 Uhr beendet werden.

Marion Bundschu, Ulm 